\title{
A woman with cystocele and rectocele struggled severe constipation
}

\section{Case report}

Chronic constipation is most common bowel complaints in women older than 60-65-year-old. The symptoms related with constipation has been increased in last 8-10years when was the period of transition time from agricultural nutrition to industrial food turnover. Constipation should be considered as a group of signs instead of disease. It has been defined as difficult, incomplete painful defecation in long interval. Two out of several criteria preceding over 3 months at least had been accepted as an adequate requirement to be diagnosed as constipation in the Rome II International consensus meeting. Those symptoms are Lumpy or hard stool, Straining, Sensation of anorectal obstruction, sensation of incomplete defecation, Fewer than 2-3 bowel movements per week, Abdominal bloating and digital maneuvering to defecate. Chronic constipation has three clinical sub-groups, slow transit time defecation (like colonic inertia), the constipation with inflammatory bowel syndrome and obstructed defecation syndrome. One of the reasons of obstructed defecation is rectocele. It has related with thin and weak rectovaginal septum where is located between posterior wall of vagina and anterior part of the rectum retroperitoneally. Although rectocele is defined as posterior vaginal wall collapse it occurs as a part of the pelvic organ prolapse (POP). However, the reason of rectocele is not clear. Weakened pelvic floor muscles have been occur in such patients. There are many reasons leading to this type of weakness including older age, vaginal deliveries and obstetric trauma like forceps delivery, vacuum delivery and episiotomy). In addition, chronic constipation with excessive straining for a long time period plays an additive role in developing of that disease. Pelvic surgeries and total hysterectomy can also influence to the weakening of the pelvic floor muscles and ligaments fixing genital organs into the pelvic bony chambers.

Cystocele, on the other hand is assessed as a part of the POP which is related with anterior vaginal wall collapse. It is most common form of female POP, often seems in elderly woman. Cardinal ligaments, lateral and fascial connections give strong support to vagina. Disruption one of these supportive structures which has been mentioned above, might precipitate POP and/or cystocele. In our practice, we have several women with rectocele complaining severe constipation what has not been solved or treated by doctors who cannot make a true diagnosis of rectocele. In this paper, the one of women with POP complaining severe constipation undergone laparoscopic sacrocolpopexy (promontofixation) has been reported. Then the short-term follow-up outcomes of those were reported.

\section{Case}

She, 67-year-old lady presenting severe constipation for several years was examined in the proctology unit. Digital rectal examination gave loose anal sphincter mechanism, large rectocele presenting a prominent Delamare's sign and total vaginal vault prolapse even little straining. She gave 7child births during her fertile period. There was no exact data about how her deliveries been happened. She underwent total hysterectomy with salphingoooferectomy12years ago. She has felt a sensation of incomplete evacuation at the end of each defecation.
Volume 5 Issue 2 - 2017

\author{
Sezai Demirbas \\ Department of General Surgery, GATA Haydarpasa Training \\ Hospital,Turkey
}

Correspondence: Sezai Demirbas, Department of General Surgery, GATA Haydarpasa Training Hospital, Istanbul, Turkey

Received: June 05, 2017 | Published: June 21, 2017

She said that she had had no valuable solution for her condition after many treatments for many years. She underwent surgery for urinary incontinence 4 years ago. She denies involuntary urination. The main complaints of her were difficult defecation, soiling and spotted underwear due to the vagina prolapsed even when she arised (Figure 1). She had several colonoscopies and rectosigmodoscopies in the past. They came all back normal outcomes without mucosal and intraluminal pathology. Endoanal ultrasonography (EAUS) had been performed to evaluate the anal canal, and lower part of the rectum. There was nothing but significant sonographic findings of both rectocele and cystocele. Internal anal sphincter (IAS) was displayed thinner than it really was. There was no imminent anatomic/pathologic defect on the external anal sphincter (EAS). Cine-defecography showed large rectocele (Figure 2). Bowel contents was not evacuated and remained in the lower part of sigmoid colon though she tried making evacuation in several attempts. In her defecography pelvic floor movement during maximal straining was displayed more than $5 \mathrm{~cm}$ beyond the pubococcygeal line. That was assessed as pelvic floor descent. Anal manotmetry evaluating the anal canal functions had been applied. Decreased anal canal pressures and higher threshold volumes of the rectum were measured as expected. Other tests done were in normal limits.

At the end of the outcomes of her tests, the patient had been diagnosed as POP with rectocele which was big ( $>5 \mathrm{~cm}$ in diameter) and located at middle-upper part of the rectum. She also had vaginal vault prolapse which was most probably due to previous surgery. Sacrocolpopexy with mesh fixation was chosen treatment for the patient with POP which could prevent excessive down movement of pelvic floor and eliminate prolapsing the vaginal vault. So functional stability of the muscles and organs at the pelvic floor arising after the surgery might benefit for rectocele. Surgical Technique; Under the general anesthesia each patient placed on lithotomy position on the table. Urinary catheterization was placed after antibiotic had been delivered. Sacrocolpopexy with 2 meshes was considered the 
surgical treatment for this lady with POP with rectocele and anal canal dysfunction. This type of surgery had been performed in selected 16women with same complaints past 5years. The surgical technique was performed by laparoscopically. Four trocars had been used. Umbilical port $(10 \mathrm{~mm})$ was for camera and two ports $(5 \mathrm{~mm})$ placed bilateral on the same line $4-5 \mathrm{~cm}$ below umbilicus. Fourth port $(5 \mathrm{~mm})$ was placed left abdominal quadrant on the anterior axillar line. Pelvic visceral peritoneum was opened to deep in the pelvis as a reverse J-shaped. The dissection around the rectum was carried out where levator ani muscles seen bilaterally deep in the pelvic floor. After this dissection, vagina was separated from bladder 3-4cm deep in the space. A Y-shaped mesh and a simple mesh $12-15 \mathrm{~cm}$ in length were put and sutured on the levator ani muscle and anterior vaginal wall. Meshes with little tension were sutured on the promontorium after 6 stiches had been placed on the meshes together. The great care gave no bleeding in the surgical site. Visceral peritoneal layers were closed by stiches to leave no mesh visible (Figure 3).
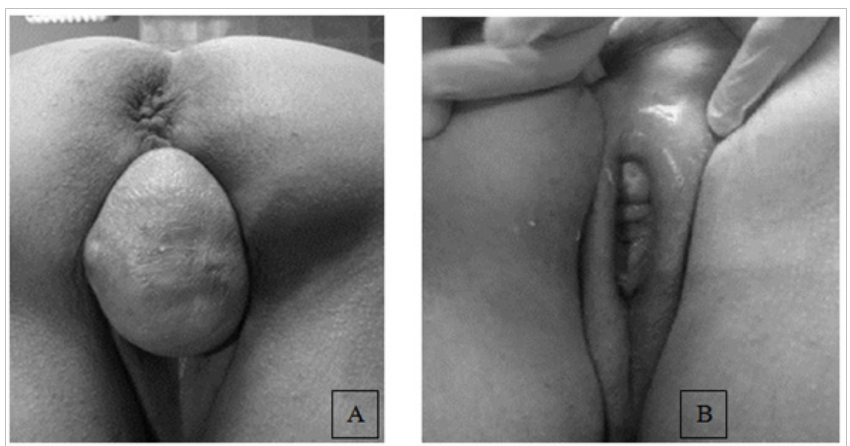

Figure 1 Total vaginal vault prolapse was seen in 1A. Dellamare sign indicating rectocele was seen in same patient $1 \mathrm{~B}$.

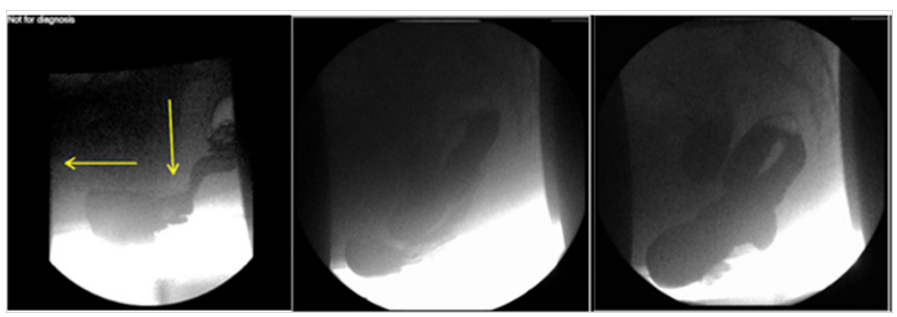

Figure 2 Rectocele clinically evident resulted to the severe constipation

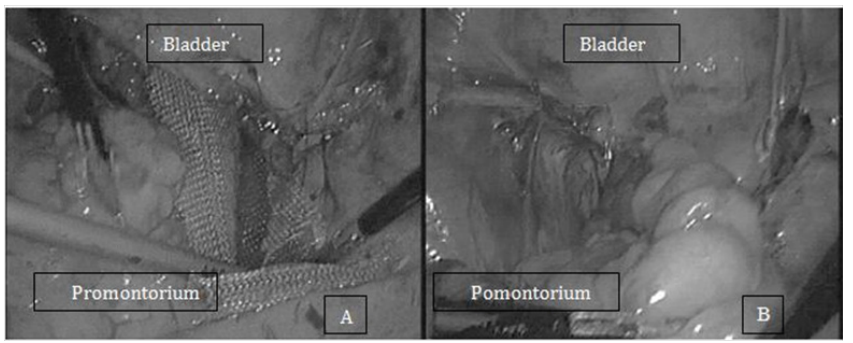

Figure 3 The upper parts of 2 meshes placed both levator ani muscle and vagina were anchored on the promontorium (A). Peritoenal layers were closed over the meshes which has no expose at the end of surgery (B).
Selected 16 patients had undergone this type of surgery using laparoscopic approach. There was no conversion to open. Some complications after surgery like trocar site bleeding $(n=1)$, urinary tract infection (3), wound infection (1), atelectasis (2). Overall complication in early postoperative period was seen in $4(25 \%)$ patients. Patients had bowel movement at postoperative day (POD) 3. They had taken oral at POD1. Hospital stay was assessed 7,25days. Antibiotic regimen was continued until POD 7 for each patient. In postoperative period, analgesic requirement was replaced by one of the non-steroid anti-inflammatory drugs. There was no need any opioid replacement 8 -10hours after surgery. All patients were followed for 16 months. At the end of 2year after surgery phone communication had been established. They were all questioned about if any recurrence was happened. At the end of that period no recurrence displayed. They were also evaluated small questionnaire asking few question about their life quality and general health. Twelve of 16 patients $(75 \%)$ described their life quality as much as good that they assumed after surgery. If necessary, they said that they would have the same surgery again. Remaining 4 individual stated no unwanted event after surgery but they felt an annoying discomfort at the pelvic area. Defecation status for each one was evaluated by some of Rome II criteria. In general, there was no constipation problem at the end of follow-up. Fecal incontinence was not a problem to be solved at that time too. At the end, laparoscopic sacrocolpopexy with two meshes (promontofixation) for a woman with POP is the way to solve both evacuation problems and descent of pelvic floor. However, during this surgery mesh has been used in the pelvic area. It is controversial. There are many debate on that subject in the literature. So, this type of surgical procedure can be applied in well-selected patients.

\section{Acknowledgements}

None.

\section{Conflict of interest}

The author declares no conflict of interest. 